\title{
Transverse field muon-spin rotation measurement of the topological anomaly in a thin film of MnSi
}

\author{
T. Lancaster, ${ }^{1, *}$ F. Xiao, ${ }^{1}$ Z. Salman, ${ }^{2}$ I. O. Thomas, ${ }^{1}$ S. J. Blundell,,${ }^{3}$ F. L. Pratt, ${ }^{4}$ S. J. Clark, ${ }^{1}$ T. Prokscha, ${ }^{2}$ A. Suter, ${ }^{2}$ \\ S. L. Zhang, ${ }^{3}$ A. A. Baker, ${ }^{3}$ and T. Hesjedal ${ }^{3}$ \\ ${ }^{1}$ Centre for Materials Physics, Durham University, Durham DH1 3LE, United Kingdom \\ ${ }^{2}$ Laboratory for Muon Spin Spectroscopy, Paul Scherrer Institut, 5232 Villigen PSI, Switzerland \\ ${ }^{3}$ Oxford University Department of Physics, Clarendon Laboratory, Parks Road, Oxford OX1 3PU, United Kingdom \\ ${ }^{4}$ ISIS Facility, STFC Rutherford Appleton Laboratory, Chilton, Didcot, Oxfordshire OX11 OQX, United Kingdom
}

(Received 12 October 2015; published 25 April 2016)

\begin{abstract}
We present the results of transverse-field muon-spin rotation measurements on an epitaxially grown 40-nmthick film of $\mathrm{MnSi}$ on $\mathrm{Si}(111)$ in the region of the field-temperature phase diagram where a skyrmion phase has been observed in the bulk. We identify changes in the quasistatic magnetic field distribution sampled by the muon, along with evidence for magnetic transitions around $T \approx 40$ and $30 \mathrm{~K}$. Our results suggest that the cone phase is not the only magnetic texture realized in film samples for out-of-plane fields.
\end{abstract}

DOI: 10.1103/PhysRevB.93.140412

There has been a flurry of recent interest in the physics of the skyrmion as an example of a topological excitation in condensed matter [1]. The simplest example of a skyrmion may be derived from a sphere studded with radially directed arrows. The skyrmion is formed via the stereographic projection of the arrows onto a plane while keeping their orientations fixed. The clearest evidence for the existence of the skyrmion is in the spin texture of magnetic systems and in recent years a number of advances have demonstrated the existence, not only of magnetic skyrmions, but also their ordering into a skyrmion lattice (SL) [2-11]. In bulk samples the SL has been observed in only a restricted region of the applied field-temperature phase diagram of magnetic systems, known as the $A$ phase. However, it has been shown that the SL phase is stabilized over an extended region of the phase diagram in bulk samples that have been thinned $[6,12]$. This motivated the search for skyrmions in epitaxially grown thin-film systems. However, the unambiguous identification of the SL in such samples has been challenging.

When a magnetic field is applied along the [111] direction below the critical temperature $T_{\mathrm{c}}^{\text {bulk }}=29.5 \mathrm{~K}$, bulk MnSi hosts four magnetically ordered phases, characterized by critical fields [2] $B_{\mathrm{c} 1} \approx 0.1 \mathrm{~T}$ and $B_{\mathrm{c} 2} \approx 0.5 \mathrm{~T}$. Below a critical field $B_{\mathrm{c} 1}$ the spins order helically with a $q$ vector parallel to the applied field; for $B_{\mathrm{c} 1}<B<B_{\mathrm{c} 2}$ the spins order conically; and when $B>B_{\mathrm{c} 2}$ the spins order ferromagnetically. In addition, for $B_{\mathrm{c} 1}<B<B_{\mathrm{c} 2}$ there exists a wedge-shaped $A$-phase region close to $T_{\mathrm{c}}^{\text {bulk }}$ (centered around $T=28 \mathrm{~K}$ and an applied field $B_{\text {app }}=150 \mathrm{mT}$ ), which hosts the SL [2,12]. As with other B20 systems when compared to the bulk, the SL was reported to exist over an extended region in thinned samples of thickness [12] $\approx 50 \mathrm{~nm}$ and also in nanowires [13]. Subsequently, a report of topological Hall effect (THE) measurements and Lorentz transmission electron microscopy (TEM) on epitaxially grown thin-film samples suggested that the skyrmion phase was significantly enlarged in field and temperature [14] as might be presumed from comparison between the phase diagrams of bulk samples and those that have been thinned. However, subsequent microscopy

\footnotetext{
*tom.lancaster@durham.ac.uk
}

work [15] challenged the notion that skyrmions are present in the thin-film samples, leading to the claim that the extended phase region responsible for the THE response was, in fact, the magnetic cone phase [16]. There have also been several experimental and theoretical investigations of thin-film MnSi [17-21] suggesting, in particular, that the ground state magnetic structure propagates along the [111] direction [18]. Further, it was suggested that in out-of-plane fields no firstorder magnetic transition is observed that would indicate the appearance of skyrmions and that the cone magnetic structure is the thermodynamically stable phase for out-of-plane applied magnetic fields with $B<B_{\mathrm{c} 2}$ [21].

In view of the controversy, there is value in using alternative experimental techniques to probe the physics of the magnetic field configurations in thin-film samples. To this end, we report the results of transverse-field muon-spin rotation (TF $\left.\mu^{+} \mathrm{SR}\right)$ measurements [22] on a thin-film sample of $\mathrm{MnSi}$. We probe the local magnetic field distribution in the region giving rise to the reported anomalous topological response in Hall measurements. Here we show that several discontinuous changes in the field distribution are observed in the $T=20-40 \mathrm{~K}$ region suggesting that the magnetic structure changes significantly, and indicating that the cone phase is unlikely to be the only magnetically ordered phase stabilized in these films.

A MnSi thin-film sample was prepared by molecular beam epitaxy on $\mathrm{Si}(111)$ substrates as described in the Supplemental Material [23]. To characterize the film, a series of static magnetization measurements was made by applying the magnetic field along the $\mathrm{MnSi}[111]$ direction. The Curie temperature $T_{\mathrm{c}}^{\text {film }}$, defined as the knee point of the $M-T$ curve [examples shown in the inset of Fig. 1(b)] is found to be $T_{\mathrm{c}}^{\text {film }}=42.3(2) \mathrm{K}$, which is consistent with the values previously reported for epitaxial MnSi films [14,17-21]. The difference between this value and that of the bulk is largely attributable to tensile strain induced by the $-3 \%$ lattice mismatch between the MnSi film and the Si substrate, as discussed previously [17]. The $M-B$ curves [Fig. 1(a)] were measured at different temperatures after field cooling from $300 \mathrm{~K}$ in an applied field of $2 \mathrm{~T}$. The Si substrate provides a large diamagnetic background at low temperatures below $T_{\mathrm{c}}$ which, along with contributions from unsaturated MnSi moments at high field [24], results 

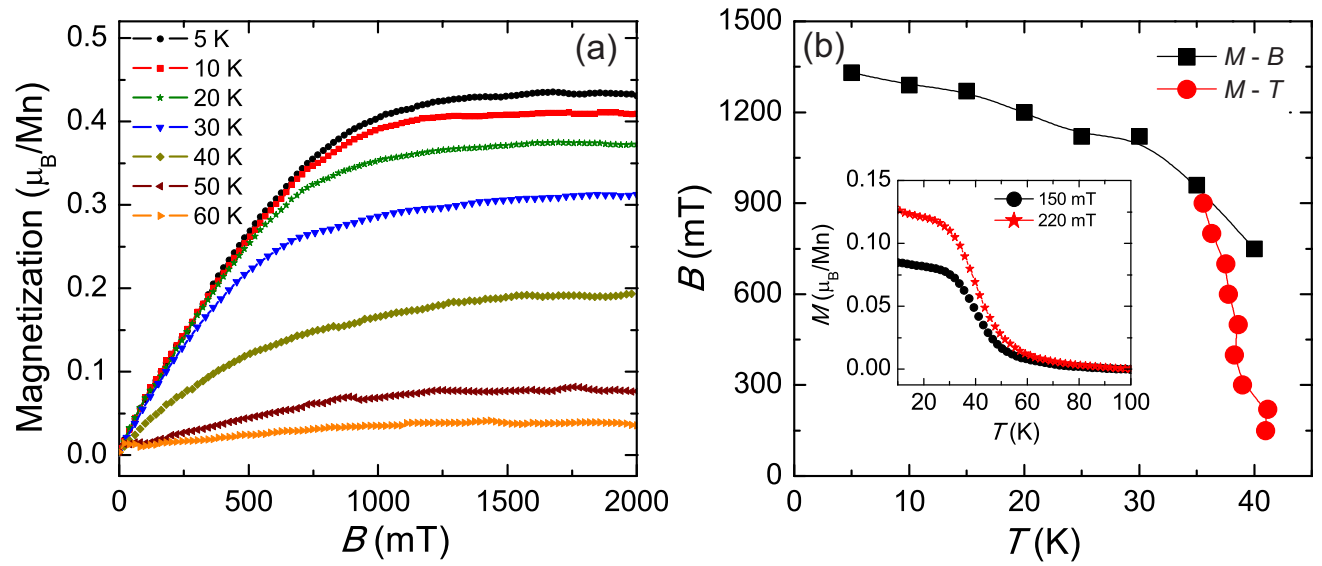

FIG. 1. Magnetometry measurements for the 40-nm-thick MnSi thin film. (a) Magnetization as a function of field at different temperatures. (b) Phase boundaries identified by $B_{\mathrm{c} 2}$ and $T_{\mathrm{c}}$. Inset: Field-cooled magnetization curve in an applied field of 150 and $220 \mathrm{mT}$.

in a linear field dependence which we subtract from the data. The saturation magnetization $M_{\mathrm{s}}$ at $5 \mathrm{~K}$ is found to be $0.41(3) \mu_{\mathrm{B}} / \mathrm{Mn}$, consistent with bulk behavior [24,25] but different from the behavior found in films that are thinner than $10 \mathrm{~nm}$ [17]. The shape of the curves is consistent with previous studies on epitaxial $\mathrm{MnSi}$ films with applied field directed out of plane [14,21]. This contrasts with field in-plane configuration (not shown), in which a sharp phase transition can be identified at lower fields in the susceptibility curves. (This effect appears to indicate that the system undergoes different evolutions of the magnetic structure in the two distinct geometries.) The kink in the hysteresis loops reported in Ref. [14] appears very weak, but can be seen in data measured between 600 and $800 \mathrm{mT}$ for 10, 20, and $30 \mathrm{~K}$.

The transition from the conical phase to the field-polarized phase at an applied field $B_{\mathrm{c} 2}$ can be extracted from the $M-B$ curves at the point where $d M / d B=0$. The values of $B_{\mathrm{c} 2}$ are found to be slightly higher than the intrinsic values reported previously, and this may be caused by demagnetization effects [17] due to the irregular sample shape used in our magnetometry measurements. Figure 1(b) shows the upper boundary of the phase diagram depicted by a series of $T_{\mathrm{c}}$ values from $M-T$ curves, and $B_{\mathrm{c} 2}$ values from $M-B$ curves, inside which the nontrivial spin textures may appear.

TF $\mu^{+}$SR measurements were made on the MnSi sample using the low energy muon beamline at $\mathrm{S} \mu \mathrm{S}$ [23,26]. Applied magnetic fields were directed perpendicular to the surface of the sample (i.e., along [111]). Our use of TF $\mu^{+} \mathrm{SR}$ to probe the SL is analogous to its use in probing the vortex lattice in a type II superconductor, where the technique provides a powerful means of measuring the internal magnetic field distribution caused by the presence of the magnetic field texture [27]. We have previously used this technique to probe the $\mathrm{SL}$ region in bulk $\mathrm{Cu}_{2} \mathrm{OSeO}_{3}$ [28]. In a TF $\mu^{+} \mathrm{SR}$ experiment, spin polarized muons are implanted in the bulk of a material in the presence of a magnetic field $B_{\text {app }}$, which is applied perpendicular to the initial muon-spin direction. Muons stop at random positions on the length scale of the field texture where they precess about the total local magnetic field $B$ at the muon site, with frequency $\omega=\gamma_{\mu} B$, where $\gamma_{\mu}=2 \pi \times 135.5 \mathrm{MHz} \mathrm{T}^{-1}$. The observed property of the experiment is the time evolution of the muon-spin polarization $P_{x}(t)$, which allows the determination of the distribution $p(B)$ of local magnetic fields across the sample volume via $P_{x}(t)=\int_{0}^{\infty} d B p(B) \cos \left(\gamma_{\mu} B t+\phi\right)$ where the phase $\phi$ results from the detector geometry.

Our TRIM.SP calculations [23] predict that an incident energy of $E=5 \mathrm{keV}$ leads to a fairly symmetrical implantation profile of muons, with a maximum $\approx 25 \mathrm{~nm}$ below the surface of the 40-nm-thick MnSi layer with FWHM $\approx 20 \mathrm{~nm}$, such that $>95 \%$ of muons are implanted in the MnSi film. TF $\mu^{+}$SR measurements were made in an applied magnetic field of $B_{\text {app }}=149 \mathrm{mT}$ after cooling in this field. This field was chosen as it is known to promote the SL phase in the bulk material. Example Fourier transform spectra, whose spectral weight is proportional to $p(B)$, measured as a function of temperature are shown in Fig. 2. At high temperatures we observe the response of muons precessing in the applied field $B_{\text {app }}=149 \mathrm{mT}$. On cooling below $60 \mathrm{~K}$ the observed line shape broadens considerably, with its mean $B_{0}$ shifting to fields lower than $B_{\text {app }}$. The line shape also becomes slightly skewed, with additional spectral weight shifting to lower fields. The line shape is seen to broaden further below the $30 \mathrm{~K} A$-phase boundary observed in the bulk, and again below $20 \mathrm{~K}$. It is notable that the spectral lines observed in our film sample seem somewhat less well resolved than those seen in a bulk sample [29]. This, along with the increased $T_{\mathrm{c}}$ observed in MnSi films, might be ascribable to strain effects in the films.

The spectra were fitted in the time domain to a polarization function $P_{x}(t)=a e^{-\sigma^{2} t^{2}} \cos \left(\gamma_{\mu} B_{0} t+\phi\right)$, where $\phi$ are phases resulting from the detector geometry, and $a$ is a fixed amplitude. The evolution of (a) the relaxation rate $\sigma=\gamma_{\mu} \sqrt{\left\langle\left(B-B_{0}\right)^{2}\right\rangle} / \sqrt{2}$, arising from those muons stopping in $\mathrm{MnSi}$, and (b) the average magnetic field $B_{0}$ that they experience, are shown in Fig. 3. The relaxation rate $\sigma$ [Fig. 3(a)] is seen to slowly increase with decreasing temperature below $100 \mathrm{~K}$, with the rate of increase becoming larger below $60 \mathrm{~K}$. There is a cusp in the curve at $40 \mathrm{~K}$, with the width remaining approximately constant in the region between 40 and $30 \mathrm{~K}$. At $30 \mathrm{~K}$, the relaxation rate $\sigma$ jumps in magnitude, and increases sharply once again below $20 \mathrm{~K}$. Although a smooth increase in $\sigma$ below $T_{\mathrm{c}}^{\text {film }}$ might be predicted on the grounds of an increase in the ordered moment of the system with decreasing 

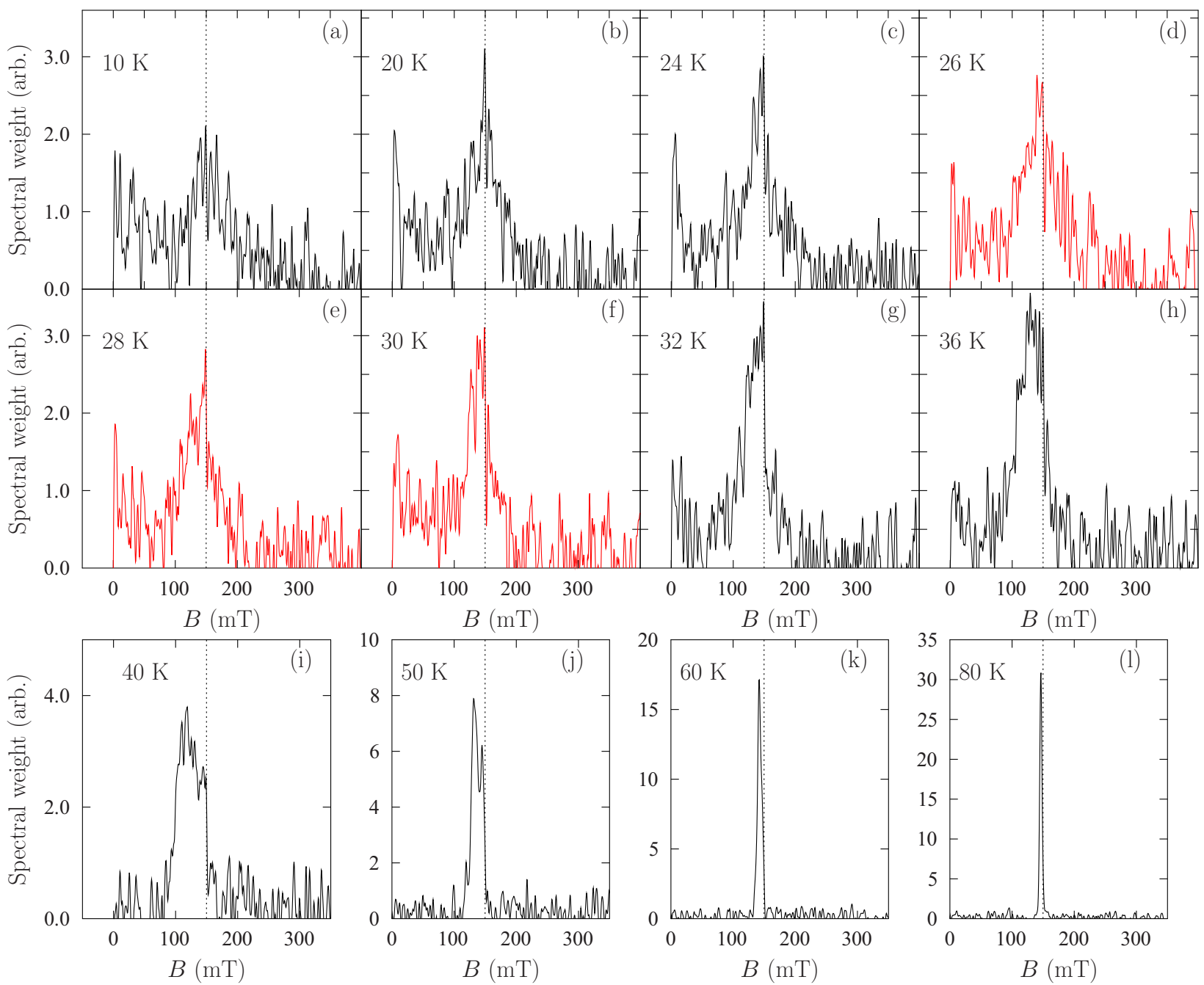

FIG. 2. Fourier transform $\mu^{+}$SR spectra measured for a MnSi film in an applied field of $B_{\text {app }}=149$ mT for muons implanted into the center of the MnSi layer in the film. The skyrmion $A$-phase region in the bulk is found between $26 \lesssim T \lesssim 30 \mathrm{~K}$ [spectra (d)-(f)]. Note the change of scale in panels (i)-(1).

temperature in the ordered phase, the observed discontinuities are not be expected in a typical ordered magnet. The average field $B_{0}$ [Fig. 3(b)] is close to the applied field in the high temperature regime around $100 \mathrm{~K}$, but is seen to decrease with decreasing temperature above $T_{\mathrm{c}}^{\text {film}}$, before reaching a sharp minimum at $40 \mathrm{~K}$. It then increases in the ordered regime until $B_{0} \approx B_{\text {app }}$ below $T \approx 30 \mathrm{~K}$.

A temperature scan carried out at a larger applied field of $220 \mathrm{mT}$ (at the same $5 \mathrm{keV}$ implantation energy) was found to show the same trends above $40 \mathrm{~K}$. The larger relaxation rates measured in these data are more difficult to fit and consequently more scatter is seen at low temperature, but the data are suggestive of similar features as those seen at $149 \mathrm{mT}$. Finally, a scan carried out for $B_{\text {app }}=149 \mathrm{mT}$, but at a muon implantation energy $E=1.3 \mathrm{keV}$ allows us to probe the response of muons implanted near to the surface of the film. In this case similar features are again seen down to $40 \mathrm{~K}$, below which the broad signal line shapes become difficult to fit [23].

Each of the features seen in the TF $\mu^{+}$SR may be correlated with those observed previously using other techniques in bulk and thin-film samples of $\mathrm{MnSi}$. We note that the general trend in $B_{0}$ above $T_{\mathrm{c}}^{\mathrm{film}}$ is accounted for by the effect of the increase in magnitude of the hyperfine coupling with decreasing temperature in the paramagnetic regime near the transition, as has previously been observed in Knight shift measurements [30]. In fact, correcting the shift for the Lorentz field and the demagnetizing field gives the Knight shift shown in the inset in Fig. 3, which when plotted against $\chi \approx \mu_{0} M / B$, measured at $B=150 \mathrm{mT}$, allows us to estimate a contact hyperfine coupling of $A_{\mathrm{HF}}=-0.95(5) \mathrm{mol} \mathrm{emu}^{-1}$, consistent with previous measurements [29].

In the bulk, the magnetic transition from the paramagnetic to the skyrmion phase occurs in these applied fields at around [31] $T_{\mathrm{c}}^{\text {bulk }}=30 \mathrm{~K}$, where we see a jump in the $\mu^{+} \mathrm{SR}$ relaxation rate $\sigma$ and change in behavior of the peak field $B_{0}$. In addition, the film ordering transition $T_{\mathrm{c}}^{\text {film }}$, identified from magnetometry and the onset of a sizable response from the THE [14], occurs at $40 \mathrm{~K}$, where we see a knee in the evolution of $\sigma$ and the maximum shift in $B_{0}$. Finally, THE measurements also show a transition or crossover below the 15-20 K region, resulting in a small or negative THE signal, which coincides 


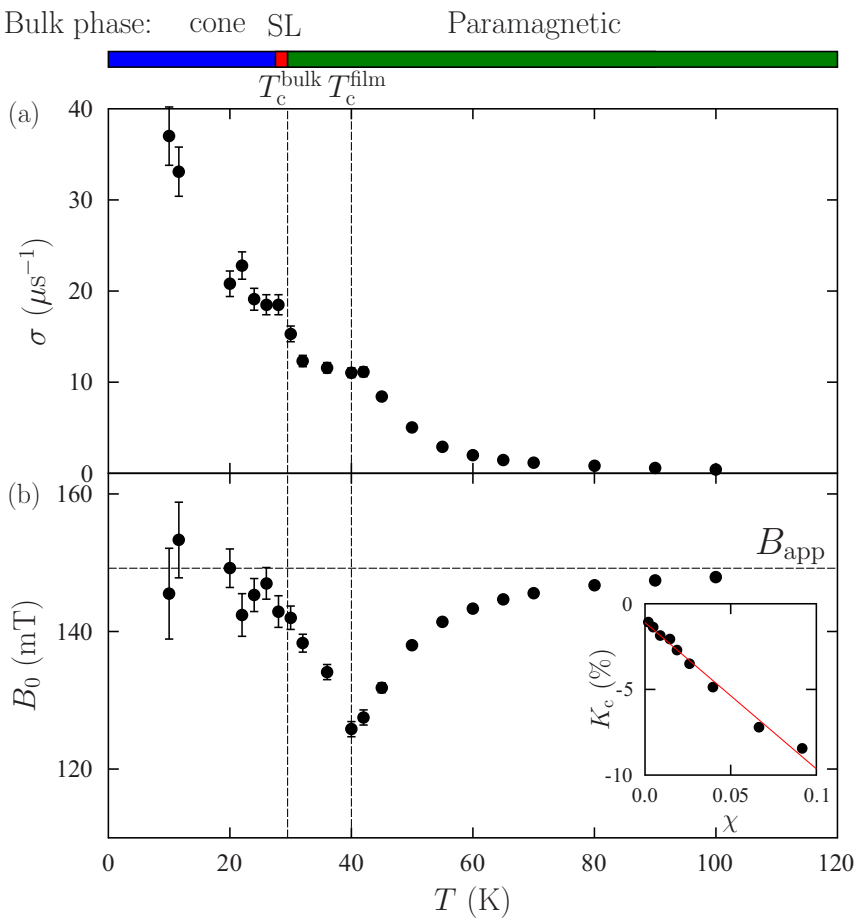

FIG. 3. Results of fitting the time domain spectra measured in an applied field of $B_{\text {app }}=149 \mathrm{mT}$ and implantation energy $E=5 \mathrm{keV}$. (a) Relaxation rate $\sigma$; (b) average magnetic field $B_{0}$. Dashed vertical lines show the positions of the critical temperatures found in films $T_{\mathrm{c}}^{\text {film }}=40 \mathrm{~K}$ and the bulk $T_{\mathrm{c}}^{\mathrm{bulk}}=29.5 \mathrm{~K}$. The bar indicates the phases found in the bulk at $150 \mathrm{mT}$. The dashed horizontal line shows $B_{\text {app }}$. The inset shows the muon Knight shift $K$ as a function of $\chi=\mu_{0} M / B$.

with a further jump in $\sigma$. We note that in the muon data the line shape broadens further still at low temperatures, possibly reflecting the increased ordered moment size.

The original interpretation of the THE data [14] was based on the assumption that a large response was the result of the scattering of carriers from a topologically nontrivial spin texture, identified with the SL phase. In view of the controversy surrounding the observation of skyrmions using Lorentz TEM [15], an alternative interpretation was suggested [16], which attributed the topological contribution to the Hall effect to additional scattering of charge carriers arising intrinsically in the magnetic cone phase. (It was also noted that the lack of any clear transition being observed in the Hall effect data prevented the signal being attributed to a separate magnetic phase [16]. However, it is also worth noting that although the signal is dominated in many films by a feature in the anomolous Hall signal that prevent the transition being observed, the transition can be seen in thinner films.) Our results suggest that the magnetic properties of this system are unlikely to be accounted for simply via effects arising from a single magnetic cone phase, but rather suggest transitions in the nature of the field distribution occurring at $T_{\mathrm{c}}^{\text {film }} \approx 40 \mathrm{~K}, T_{\mathrm{c}}^{\text {bulk }} \approx 30 \mathrm{~K}$, and possibly below $20 \mathrm{~K}$. Since the $T=30$ and $20 \mathrm{~K}$ features do not have a counterpart in the magnetization measurements, they presumably do not involve a sizable rearrangement of the net component of the magnetization along the field direction.
However, the changes in muon line shape, reflected in the linewidth $\sigma$ and static field shift in $B_{0}$, that we observe below $T_{\mathrm{c}}^{\mathrm{film}}$ are suggestive of changes in the distribution of magnetic fields at the muon sites. This could suggest sizable changes to the contact hyperfine fields or to the nature of the dynamics. In the case of the latter, for example, a freezing of relaxation channels in the fast-fluctuation limit could lead to an increase in $\sigma$ on cooling. However, given the change in line shape with temperature, the shift in $B_{0}$ in the $30 \leqslant T \leqslant 40 \mathrm{~K}$ region, and the fact that a transition around $30 \mathrm{~K}$ is known to occur in the bulk, we believe the most likely explanation is that the features observed imply changes in the ordered spin structure (which itself would likely also involve a change in hyperfine fields and dynamics).

To assess the evidence for the existence of skymions in this system, we have carried out simulations of the predicted muon line shape in order to compare the expected signal for a cone, helical, and skyrmion lattice phase. The local magnetic field distributions were generated following the procedure outlined in Ref. [28]. We generate helical and conical spin textures with propagation vectors oriented along the [111] direction. The SL is generated with the skyrmion lattice plane perpendicular to [111]. Helical and SL distributions are generated as described previously [28], while cone-phase distributions are generated using [21] $\boldsymbol{m}(\boldsymbol{r}) / \boldsymbol{m}_{0}=\hat{\boldsymbol{x}} \sin \theta \cos$ $(\boldsymbol{q} \cdot \boldsymbol{r})+\hat{\boldsymbol{y}} \sin \theta \sin (\boldsymbol{q} \cdot \boldsymbol{r})+\hat{z} \cos \theta$, where $\cos \theta=|B| / B_{\mathrm{c} 2}$ and $\boldsymbol{B}\|\hat{\boldsymbol{z}}\|[111]$. The magnetic moment is taken to be $0.4 \mu_{B}$ [32], $B_{\mathrm{c} 2}=0.5 \mathrm{~T}$ (corresponding to $T_{\mathrm{c}}^{\mathrm{film}} \approx 40$ $\mathrm{K}$ [21]) and the length scale of the helical [29] and conical structures is taken to be $18 \mathrm{~nm}$. For the skymion lattice we consider lattice constants suggested in previous studies of both $L_{\mathrm{sk}}=18 \mathrm{~nm}$ [12] and $8.5 \mathrm{~nm}$ [14], although there is little difference between the two. We evaluate dipole fields from the moment distributions along with the contribution from the contact hyperfine field, calculated using [29] $B_{c}=$ $\frac{4 V_{\mathrm{mol}} A_{\mathrm{HF}}}{N V_{\text {cell }}} \sum_{j=1}^{N} \boldsymbol{m}\left(\boldsymbol{r}_{j}\right)$, where $V_{\mathrm{mol}}$ is the molar volume of $\mathrm{Mn}$ ions, $V_{\text {cell }}$ is the unit cell volume, $A_{\mathrm{HF}}=-0.9276 \mathrm{~mol} / \mathrm{emu}^{-1}$ is the hyperfine coupling from Ref. [29], and $N$ is the number of $\mathrm{Mn}$ ions within one lattice constant of the muon site. The field distribution $p(B)$ sampled by the muon ensemble has been shown to arise from muons stopped in the $4 a$ Wyckoff position. These give rise to two magnetically distinct classes of site, conventionally labeled sites 0 and 1 [29].

The predicted field distributions $p(|B|)$ in an applied field of $B_{\text {app }}=150 \mathrm{mT}$, shown in Figs. 4(a) and 4(b), are seen to be quite distinct in each phase. The cone magnetic structure [Fig. 4(a)] involves a distribution of fields (resulting from muons at site 1) and a sharp peak on the low field side of the applied field (resulting from site 0). The signal from the skyrmion magnetic structure [Fig. 4(b)] shows significant broadening over that resulting from the cone and helical magnetic structures. The shape remains asymmetric, but is less skewed than the distribution expected from the conical structure. In addition, the contribution from site 0 shows an asymmetric distribution, with a sharp peak on the low field side of the applied field. The features in the simulations are not apparent in the measured data, which also includes, for example, a background contribution from muons stopping in the sample holder. We note, however, that spectra measured in 

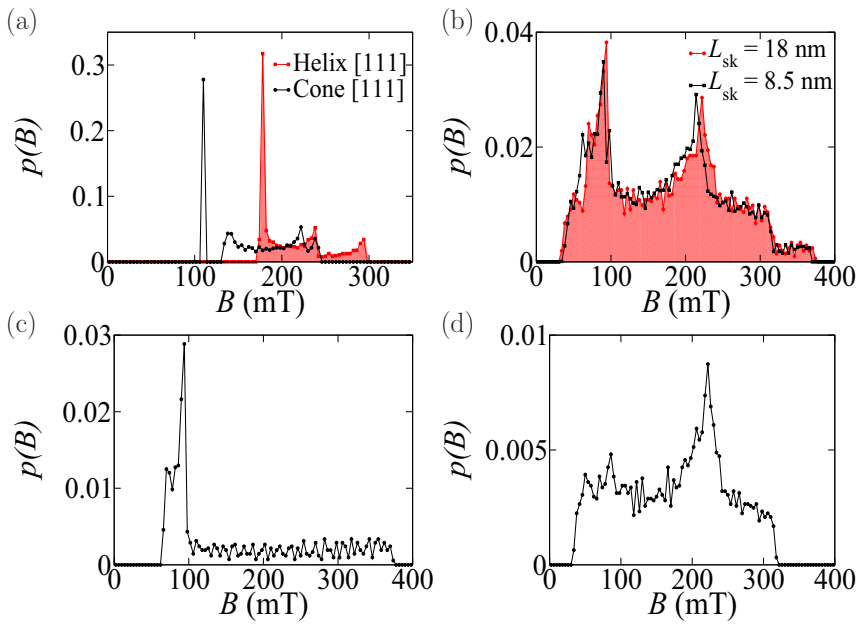

FIG. 4. The $p(B)$ distribution in an applied field of $B_{\mathrm{a}}=150 \mathrm{mT}$. (a) Helical and conical magnetic structures. (b) The $p(B)$ distribution for the SL magnetic structure with lattice constants $L_{\mathrm{sk}}=8.5$ and $18 \mathrm{~nm}$. (c),(d) Field distributions at stopping sites 0 and 1, respectively, for the SL structure with $L_{\mathrm{sk}}=18 \mathrm{~nm}$.

the region where the SL phase is observed in bulk MnSi $(26 \lesssim$ $T \lesssim 30 \mathrm{~K}$ ) do show a broadened field distribution compared to those measured in the $30 \lesssim T \lesssim 40 \mathrm{~K}$ region, with some spectral weight shifted to fields below the diamagnetic peak. It is also noticeable that $p(B)$ for the cone structure involves a sizable shift of the peak in spectral weight to low fields and that, in the data, the value of the field $B_{0}$ recovers towards the applied field below $40 \mathrm{~K}$ with the shift no longer apparent below $T=30 \mathrm{~K}$. Despite these observations, it is not possible from the comparison of these simulations to the measured line shapes of Fig. 2, to make firm conclusions regarding the nature of the spin structure in the films, or to unambiguously conclude whether skyrmions are present. Whatever the case, it is unlikely that the changes that we see in the $T=20-40 \mathrm{~K}$ region can be accounted for simply by a single magnetic cone phase. It is also unlikely that our measurements could be explained via a single magnetic phase with the formation of chiral domains of the form discussed, e.g., in Ref. [33]. In that case, we would expect the muon response in the two domains to be different (based on the behavior of the bulk material [29]), resulting in a broadening compared to the single domain case. However this would not, in itself, explain the succession of changes in broadening we observe on cooling.

In conclusion, magnetization and $\mathrm{TF} \mu^{+} \mathrm{SR}$ measurements in thin-film MnSi identify an ordering temperature at $T_{\mathrm{c}}^{\text {film }} \approx$ $40 \mathrm{~K}$. The TF data also reveal significant changes in the static field distribution that coincide with the topological contribution to the Hall effect identified previously, and also with the magnetic phase boundaries observed in both the bulk and thin films. We therefore suggest that there may be phase boundaries or crossovers in behavior in this system occurring around $T \approx 20,30$, and $40 \mathrm{~K}$. Although our data do not reveal a signature of the SL phase, it is unlikely that a single cone phase could account for our results.

This work was carried out at $\mathrm{S} \mu \mathrm{S}$, Paul Scherrer Institut, Switzerland and we are grateful for the provision of beam time. We thank the EPSRC (UK) and the John Templeton Foundation for financial support and to A. Amato, Ch. Pfleiderer, and R. C. Williams for useful discussion. S.L.Z. and T.H. gratefully acknowledge support by the Semiconductor Research Corporation (SRC). This work made use of the facilities of N8 HPC provided and funded by the N8 consortium and EPSRC (Grant No. EP/K000225/1). The Centre is coordinated by the Universities of Leeds and Manchester.

Data presented in this paper will be made available via http://dx.doi.org/10.15128/5q47rn72z.
[1] The Multifaceted Skyrmion, edited by G. E. Brown and M. Rho (World Scientific, Singapore, 2010).

[2] S. Mühlbauer, B. Binz, F. Jonietz, C. Pfleiderer, A. Rosch, A. Neubauer, R. Georgii, and P. Böni, Science 323, 915 (2009).

[3] W. Münzer, A. Neubauer, T. Adams, S. Mühlbauer, C. Franz, F. Jonietz, R. Georgii, P. Böni, B. Pedersen, M. Schmidt, A. Rosch, and C. Pfleiderer, Phys. Rev. B 81, 041203(R) (2010).

[4] X. Z. Yu, Y. Onose, N. Kanazawa, J. H. Park, J. H. Han, Y. Matsui, N. Nagaosa, and Y. Tokura, Nature (London) 465, 901 (2010).

[5] X. Z. Yu, N. Kanazawa, Y. Onose, K. Kimoto, W. Z. Zhang, S. Ishiwata, Y. Matsui, and Y. Tokura, Nat. Mater. 10, 106 (2010).

[6] S. Seki, X. Z. Yu, S. Ishiwata, and Y. Tokura, Science 336, 198 (2012).

[7] S. Seki, J.-H. Kim, D. S. Inosov, R. Georgii, B. Keimer, S. Ishiwata, and Y. Tokura, Phys. Rev. B 85, 220406(R) (2012).

[8] S. Seki, S. Ishiwata, and Y. Tokura, Phys. Rev. B 86, 060403 (2012).
[9] M. C. Langner, S. Roy, S. K. Mishra, J. C. T. Lee, X. W. Shi, M. A. Hossain, Y.-D. Chuang, S. Seki, Y. Tokura, S. D. Kevan, and R. W. Schoenlein, Phys. Rev. Lett. 112, 167202 (2014).

[10] T. Adams, A. Chacon, M. Wagner, A. Bauer, G. Brandl, B. Pedersen, H. Berger, P. Lemmens, and C. Pfleiderer, Phys. Rev. Lett. 108, 237204 (2012).

[11] A. A. Omrani, J. S. White, K. Prša, I. Živković, H. Berger, A. Magrez, Y.-H. Liu, J. H. Han, and H. M. Rønnow, Phys. Rev. B 89, 064406 (2014).

[12] A. Tonomura, X. Yu, K. Yanagisawa, T. Matsuda, Y. Onose, N. Kanazawa, H.-S. Park, and Y. Tokura, Nano Lett. 12, 1673 (2012).

[13] X. Yu, J. P. DeGrave, Y. Hara, T. Hara, S. Jin, and Y. Tokura, Nano Lett. 13, 3755 (2013).

[14] Y. Li, N. Kanazawa, X. Z. Yu, A. Tsukazaki, M. Kawasaki, M. Ichikawa, X. F. Jin, F. Kagawa, and Y. Tokura, Phys. Rev. Lett. 110, 117202 (2013).

[15] T. L. Monchesky, J. C. Loudon, M. D. Robertson, and A. N. Bogdanov, Phys. Rev. Lett. 112, 059701 (2014). 
[16] S. A. Meynell, M. N. Wilson, J. C. Loudon, A. Spitzig, F. N. Rybakov, M. B. Johnson, and T. L. Monchesky, Phys. Rev. B 90, 224419 (2014).

[17] E. A. Karhu, S. Kahwaji, T. L. Monchesky, C. Parsons, M. D. Robertson, and C. Maunders, Phys. Rev. B 82, 184417 (2010).

[18] E. A. Karhu, S. Kahwaji, M. D. Robertson, H. Fritzsche, B. J. Kirby, C. F. Majkrzak, and T. L. Monchesky, Phys. Rev. B 84, 060404 (2011).

[19] E. A. Karhu, U. K. Rößler, A. N. Bogdanov, S. Kahwaji, B. J. Kirby, H. Fritzsche, M. D. Robertson, C. F. Majkrzak, and T. L. Monchesky, Phys Rev. B 85, 094429 (2012).

[20] M. N. Wilson, E. A. Karhu, D. P. Lake, A. S. Quigley, S. Meynell, A. N. Bogdanov, H. Fritzsche, U. K. Rößler, and T. L. Monchesky, Phys. Rev. B 88, 214420 (2013).

[21] M. N. Wilson, A. B. Butenko, A. N. Bogdanov, and T. L. Monchesky, Phys. Rev. B 89, 094411 (2014).

[22] S. J. Blundell, Contemp. Phys. 40, 175 (1999).

[23] See Supplemental Material at http://link.aps.org/supplemental/ 10.1103/PhysRevB.93.140412 for details of sample preparation, experimental methods, and further data.

[24] D. Bloch, V. Jaccarino, J. Voiron, and J. H. Wernick, Phys. Lett. A 51, 259 (1975).

[25] M. Lee, Y. Onose, Y. Tokura, and N. P. Ong, Phys. Rev. B 75, 172403 (2007).
[26] T. Prokscha, E. Morenzoni, K. Deiters, F. Foroughi, D. George, R. Kobler, A. Suter, and V. Vrankovic, Nucl. Instrum. Methods Phys. Res., Sect. A 595, 317 (2008).

[27] J. E. Sonier, J. H. Brewer, and R. F. Kiefl, Rev. Mod. Phys. 72, 769 (2000).

[28] T. Lancaster, R. C. Williams, I. O. Thomas, F. Xiao, F. L. Pratt, S. J. Blundell, J. C. Loudon, T. Hesjedal, S. J. Clark, P. D. Hatton, M. Ciomaga Hatnean, D. S. Keeble, and G. Balakrishnan, Phys. Rev. B 91, 224408 (2015).

[29] A. Amato, P. Dalmas de Réotier, D. Andreica, A. Yaouanc, A. Suter, G. Lapertot, I. M. Pop, E. Morenzoni, P. Bonfà, F. Bernardini, and R. De Renzi, Phys. Rev. B 89, 184425 (2014).

[30] R. S. Hayano, Y. J. Uemura, J. Imazato, N. Nishida, K. Nagamine, T. Yamazaki. Y. Ishikawa, and H. Yasuoka, J. Phys. Soc. Jpn. 49, 1773 (1980).

[31] A. Bauer and Ch. Pfleiderer, Phys. Rev. B 85, 214418 (2012).

[32] K. Moyoya, H. Yasuoka, Y. Nakamura, V. Jaccarino, and J. H. Wernick, J. Phys. Soc. Jpn. 44, 833 (1979).

[33] N. A. Porter, P. Sinha, M. B. Ward, A. N. Dobrynin, R. M. D. Brydson, T. R. Charlton, C. J. Kinane, M. D. Robertson, S. Langridge, and C. H. Marrows, arXiv:1312.1722. 